\title{
Teaching and Learning in Statistics: Harnessing the power of modern statistical software to improve students statistical reasoning and thinking
}

\author{
Clair L. Alston-Knox ${ }^{1}$, Christopher M. Strickland ${ }^{1}$, Theo Gazos ${ }^{1}$, Kerrie L. \\ Mengersen $^{1,2}$ \\ ${ }^{1}$ AutoStat Institute. Level 7, Melbourne VIC, Australia. ${ }^{2}$ Queensland University of \\ Technology, QLD, Australia
}

\begin{abstract}
The reproducibility crisis in science has launched global discussion about the need to restructure the way statistics is taught across a wide range of disciplines. While this need has been recognized and discussed in the academic community for many years, the impetus for educational reform of statistics was boosted by Ioannidis (2005), which resulted in a great deal of attention on issues regarding the inappropriate use of statistical reasoning.

The availability of data across business and research has increased dramatically in recent years. This access to data has resulted in almost every member of society needing a skill set that allows them to think critically about the inferences that can validly be drawn to improve decisions based on data.

One way of improving statistical literacy and thinking is through the identification and use of appropriate statistical software that will allow students, and other practitioners with basic training, access to modern statistical modeling techniques on a platform that allows them to focus on outcomes. A key component of using AutoStat for teaching statistical thinking is in alleviating the need for coding, which allows the instructors to focus on key concepts, questions and outcomes.
\end{abstract}

Keywords: statistical education; data literacy; statistical software; AutoStat; student engagement. 
Teaching and Learning in Statistics: Harnessing the power of modern statistical software to improve students statistical reasoning and thinking

\section{Introduction}

Ioannidis (2005), brought to the fore a long debated issue in research, which is now commonly referred to as the "replication crisis in science", which has inspired a number of subsequent publications (Fraser \& Reid, Gelmen \& Loken 2014) emphasizing the role that statistics, and in particular, statistical education, has played in the current dilemma.

Since the publication of this pivotal paper (Ioannidis, 2005), an outpouring of passionate views in terms of statistical education across many disciplines has seemingly culminated in a concerted effort to modernize statistical training, often termed "statistics education". This sentiment is not new, for example, George Cobb has long championed the need to reform the teaching of statistics (Cobb, 1992, 1993), and many professional meetings during the 1990's, such as the 1992 Committee on Applied and Theoretical Statistics (CATS) workshop on "Modern Interdisciplinary University Statistics Education" noted that the content of many undergraduate courses lagged behind the current recommended best practices in statistics at a concerning level. It seems, however, little change in introductory courses has taken place, with Cobb (2015) arguing that the undergraduate curriculum in statistics now needs a complete overhaul.

The demographics of students requiring statistical training has become extremely diverse. It is now generally accepted that the collection of data in modern society is so abundant, that almost everyone requires a solid grounding in statistical thinking. As noted in Deng (2015), however, the ability of people to understand data has been far outstripped by their ability to access data. It is from this position that many courses in data science, introductory statistics and other research fields that have a lower level of contribution from quantitative analysis, may benefit from exposing students to statistical concepts through the use of software designed with this purpose in mind.

Further to this, statistics education in the modern era needs to be shifted from the traditional math and science subjects and evolve into a subject that is accessible to students training in a wide variety of disciplines (Zieffler et al. 2018). The needs of students in various fields differ, but it is a general consensus that modern statistical education should provide statistical literacy, statistical reasoning and statistical thinking as a basis for a positive learning outcome (Steel et al. 2019). Cobb (2015) reiterates the approach proposed by Brown and Kass (2009), calling for undergraduate courses in statistics that make the fundamental concepts of statistical thinking accessible to a wide range of students, in various disciplines, and to minimize the mathematical prerequisites for general courses. One way to achieve this outcome is by using computing to explain and discuss concepts and inferences that can be drawn from data analysis. 


\section{Teaching statistics using modeling}

While there has been a great deal of angst towards Null Hypothesis Significance Testing (NHST) and it's contribution to the current reproducibility crisis (ASA statement 2016), perhaps the simplest way forward is to introduce a teaching culture that by-passes the use of $\mathrm{p}<0.05$ and the testing mentality that is so often used in introductory statistics courses. As noted by Rodgers (2010), practicing statisticians moved from NHST towards modeling with the availability of computers becoming commonplace. Indeed, statisticians themselves no longer view the practice of statistics as applying a set of mechanical procedures and conducting decisions in regards to $\mathrm{p}<0.05$, but rather as a process of building models and assessing their merit. If introductory statistical courses allowed students to start in the modeling space, we believe that the learning outcomes would be enhanced, and the tendency to make their own research data fit into a set of narrowly focused procedures would dissipate.

The importance of modeling and its natural role in assisting students to develop the skills of statistical reasoning is also noted in the call for papers for The Eleventh International Research Forum on Statistical Reasoning, Thinking, and Literacy. This overarching statement for the meeting makes clear that this groups perception is that by allowing students to focus on the process of modeling, rather than the actual model itself, it will encourage them to rethink the role of statistics which will encourages a better understanding of data and chance.

Moving forward, from a teaching perspective, replacing the standard NHST based curriculum with a model based approach, will see its success hinge on choosing an appropriate software platform. To obtain learning outcomes that improve students statistical thinking, choosing the correct software will allow students to focus more on the variety of outputs of the model and data analysis, thus facilitating greater investment in the problem solving, reasoning and decision making aspects of statistics.

\section{The Choice of AutoStat in Teaching}

Statistical Rethinking (McElreath, 2016) is an example of a modern statistical course that places an emphasis on statistical models, using computers and coding to understand the models and algorithms, rather than a strictly mathematical approach. While McElreath (2016) uses R and Stan, which he argues increases students statistical thinking by the implementation of the models via command line coding, others, such as Peng (2015) argue the need for statistical software that can assist researchers who will only receive basic training in statistics to produce robust, reproducible research outcomes. 
Teaching and Learning in Statistics: Harnessing the power of modern statistical software to improve students statistical reasoning and thinking

While R and similar scripting based software can aid statistical learning for some students, they are frequently studying STEM streams. In particular, for students of statistics, coding is an excellent means of understanding the algorithm behind the model. However, notwithstanding the great advances in operability of these platforms, many students - and researchers - do not have the interest, time, skills, repeated exposure or inclination to overcome the learning step to fully access the power and utility of the packages and keep abreast of updates. This can result in students and researchers feeling excluded or frustrated, withdrawing their initial interest in statistics and even becoming resistant to the topic. Moreover, even if they overcome these challenges, they can still be confronted by the task of ensuring that their adopted methods are appropriate and reproducible.

AutoStat is a new statistical software package that embeds state-of-the-art statistical and machine learning tools in an accessible, modeling-focused interface. It is designed to meet the needs of researchers and students who would otherwise be excluded from the process of modern statistical modeling due to their lack of coding acumen or through an academic inclination that does not encompass coding. It also addresses the need for appropriate, reproducible statistical approaches by removing many of the error-prone steps associated with coding, choice of algorithm and presentation of results. The look and feel of AutoStat, combined with the addition of both short and long tutorials, specialized modules for common analyses and auto predict options, is designed to facilitate confidence in modeling and further exploration of statistical paradigms and potential.

Many traditionally non-mathematical fields of study are increasingly requiring statistical studies to be incorporated in their course syllabus. However, as these topics are likely to be a minor inclusion in the overall scheme of the degree, it is essential that these students will be taught statistical thinking and concepts, rather then the mechanics of computing. For example, in the field of Social Sciences, leading academics (Jackman 2009, Kaplan 2014, van de Schoot et al. 2013) have recently presented strong arguments for the practicality of using Bayesian analysis for modeling and answering many research questions in this field. However, access to Bayesian statistical methods for both teaching and research in Social Sciences is restricted by the availability of suitable software for this audience. Similarly, modern statistical machine learning methods are essential for modern data analysts and researchers in the 'big data' era, but are often inaccessible in standard software. Rather than treating Bayesian and Frequentist paradigms as disparate entities or statistics and machine learning as separate disciplines, AutoStat switches seamlessly between them. This enables students and researchers to better understand the similarities and differences between the various approaches and underlying philosophies, and allows instructors to teach concepts and thinking, as opposed to coding. 


\subsection{The advantage of the logical flow of AutoStat}

A key feature of AutoStat for teaching is the logical flow provided in the user interface. The sequence provided in AutoStat begins with the Project Menu (Figure 1), where students will be able to organize their projects, and collaborate through the share facility when doing group work. The Task Menu (Figure 2) then provides a clear set of procedures available within the software, namely data management, visualization, model building, result summaries and report builders.

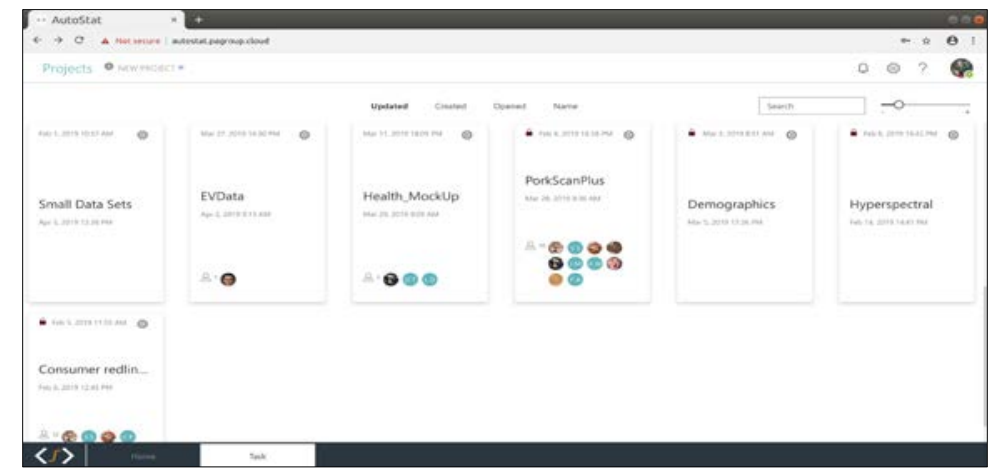

Figure 1.The Project Menu shows all current projects and members. Project sharing is allocated by the project owner, who may elect to share a copy and nominate sharing permissions. The account administrator can restrict access to non-required modeling or other modules to prevent account overuse.

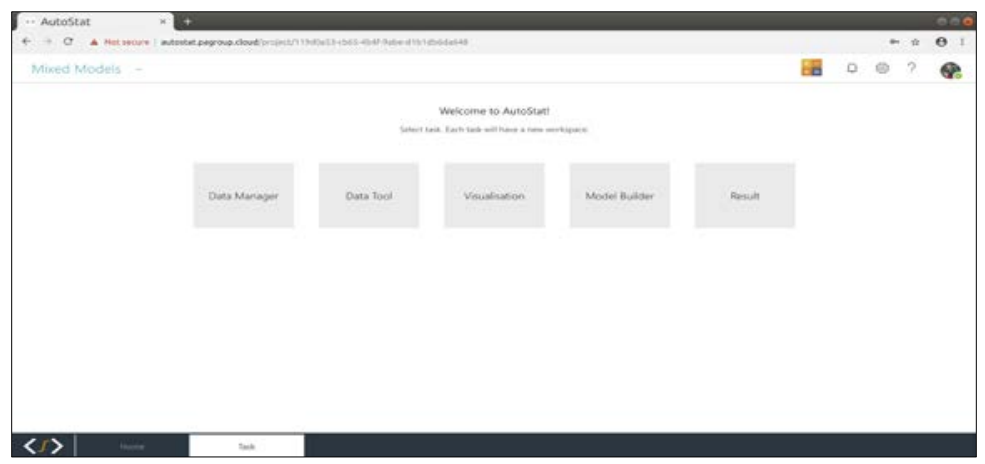

Figure 2. The Task Menuallows students to understand the logical flow from data management through to visualization as a exploratory data analysis tool, then through to modeling and interpretation of results. NHST is available within the Data Tool, a placement which emphasizes its role more along the lines of exploratory analysis and encourages further exploration via modeling.

The inbuilt data management facilities allow users to import their data and perform all necessary adjustments, such as factorization and filtering by the use of a drop down menu. The flow of the Task Menu is deliberate, in that the left to right setting of the options 
Teaching and Learning in Statistics: Harnessing the power of modern statistical software to improve students statistical reasoning and thinking

implies the logical flow of statistical analysis, namely the following 5 steps (where steps 3 and 4 are an iterative process):

1. Data management

2. Visualization

3. Statistical modeling

4. Examination of results

5. Reporting outcomes

These steps are conducted quite easily by the student using drop down or drag and drop features in the GUI (See Figures 3 \& 4). This style of GUI allows the student to perform their statistical analysis in the familiar drag and drop with either a mouse, or more casually on touch screens, such as an IPad or tablet device.

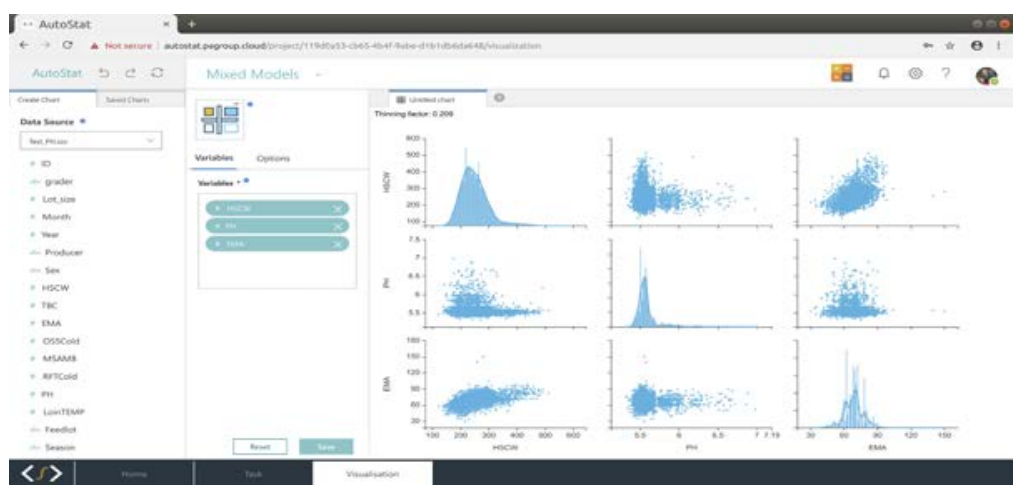

Figure 3. Visualizations are available using drag and drop via a mouse or touchscreen

An advantage of touchscreen drag and drop while teaching concepts is the ease with which the model is constructed. In this way, very little intellectual resources are diverted towards "making the computer work", and this allows the instructor to "invite your students to stay with the question” (Stangl, 2017).

Importantly, AutoStat has been devised to aid in the reproducibility of research (or model based decisions) for students well into their future careers, whether they be research based, or industry related. The logical flow of work, accessible data management and queries, ready access to stored results from all models in the history of the project and inbuilt report editor are helpful to people with only basic training, tending to assist in the pipeline of logical thinking of data outcomes. AutoStat incorporates modern algorithms, which are intended to be kept up to date with statistical developments, with older techniques being phased out of the model builder when appropriate, yet remaining available to re-run past analyses when required. 


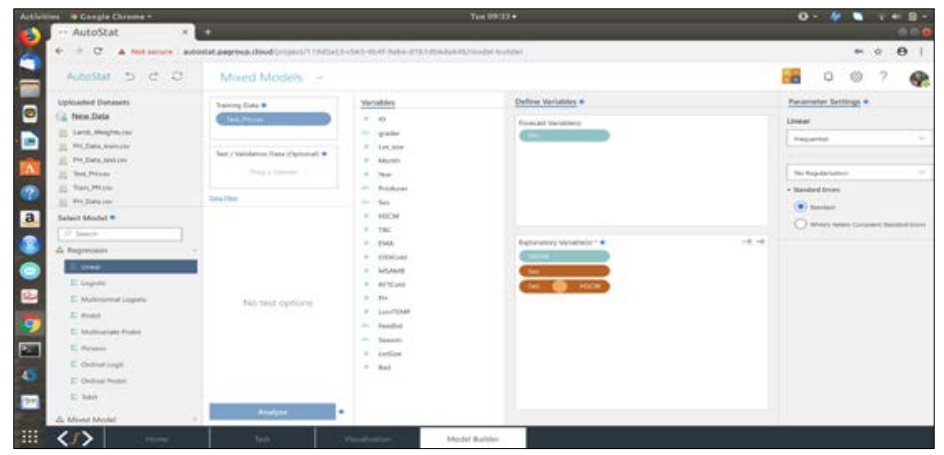

Figure 4: Modeling is performed using drag and drop options

\section{Discussion}

The current debate around research reproducibility has highlighted the need for new and novel approaches to increasing data literacy and statistical thinking among a broad range of students and professions. Indeed, as suggested by Peng (2015), increasing the data literacy and thinking skills across a range of student study streams will assist ease these current issues by improving the quality of research papers, and hence reducing the pressure on the peer review system. The provision of statistical software that is accessible to a range of users is an essential element of overhauling the current educational approach to statistical learning.

AutoStat has the potential to free students' intellectual resources in order to focus on understanding the ideas, concepts and inferences in statistical modeling, and will allow them to apply modern, innovative statistical methods to their own research into the future.

The prevalence of data in modern society has also fueled this need, with a broad range of people requiring an education that allows them to make decisions based on thinking critically in regards to available data sources. While many people attempting to make decisions based on data have the ability, with access to appropriate training, to understand statistical concepts and think in these terms, performing such tasks in the real world will be contingent upon using a software that is appropriate for their frequency of use.

\section{References}

Brown, E. N. \& Kass, R. E. (2009), “What is Statistics?”, The American Statistician, 63, 105-110.

Cobb, G. W. (1992), “Teaching Statistics: More Data, Less Lecturing,” in Heeding the Call for Change, ed. Lynn Steen, MAA Notes No. 22, p. 3-43, Washington, D.C.: Mathematical Association of America. 
Teaching and Learning in Statistics: Harnessing the power of modern statistical software to improve students statistical reasoning and thinking

Cobb, G. W. (1993), “Reconsidering Statistics Education: A National Science Foundation conference," Journal of Statistics $\quad$ Education, 1. www.amstat.org/publications/jse/v1n1/cobb.html.

Cobb, G. W. (2015) Mere Renovation is Too Little Too Late: We Need to Rethink our Undergraduate Curriculum from the Ground Up, The American Statistician, 69:4, 266282, DOI: 10.1080/00031305.2015.1093029

Fraser DAS, \& Reid N. (2016). Crisis in science? Or crisis in statistics! Mixed messages in statistics with impact on science. Journal of Statistical Research, 48-50 (1), 1-9.

Gelman, A, \& Loken, E. (2014). The Statistical Crisis in Science Data Dependent Analysis - a "Garden of Forking Paths"- Explains Why Many Statistically Significant Comparisons Don’t Hold Up. American Scientist 102(6):460.

Ioannidis, J. (2005). Why Most Published Research Findings Are False. PLoS Medicine, 2(8), 696-701. https://doi.org/10.1371/journal.pmed.0020124

Jackman, Simon (2009). Bayesian analysis for the social sciences. Wiley, Chichester, U.K

Kaplan, D. (2014). Methodology in the social sciences. Bayesian statistics for the social sciences. New York, NY, US: Guilford Press.

McElreath, R. (2016). Statistical rethinking: A Bayesian course with examples in $R$ and Stan. Boca Raton, FL, USA: CRC Press.

Peng, R. (2015), The reproducibility crisis in science: A statistical counterattack. Significance,12:30-32.doi:10.1111/j.1740-9713.2015.00827.x

Rodgers, J. L. (2010). The epistemology of mathematical and statistical modeling: A quiet methodological revolution. American Psychologist, 65(1), 1-12. http://dx.doi.org/10.1037/a0018326

Stangl, D (2017). Urging paradigm change. In Session: What Must Change in the Teaching of Statistical Inference in Introductory Courses? ASA Symposium on Statistical Inference, Bethesda. October 11, 2017- October 13, 2017.

Steel, E.A, Liermann, M. \& Guttorp , P.(2019) Beyond Calculations: A Course in Statistical Thinking, The American Statistician, 73:sup1, 392-401, DOI: 10.1080/00031305.2018.1505657.

van de Schoot R, Kaplan D, Denissen J, Asendorpf JB, Neyer FJ, van Aken MAG. A gentle introduction to Bayesian analysis: applications to developmental research. Child Dev. 2013;85(3):842-860.

Zieffler A., Garfield J., Fry E. (2018) What Is Statistics Education?. In: Ben-Zvi D., Makar K., Garfield J. (eds) International Handbook of Research in Statistics Education. Springer International Handbooks of Education. Springer 